\title{
Dietary patterns and incident cardiovascular disease in Scottish adults: a data linkage study
}

\author{
L.C.A. Craig ${ }^{1}$ and G. McNeill ${ }^{1,2}$ \\ ${ }^{1}$ Rowett Institute of Nutrition and Health and ${ }^{2}$ Institute of Applied Health Sciences, University of Aberdeen, Aberdeen, \\ $A B 252 Z D, U K$
}

A diet rich in fruit and vegetables, oily fish, fibre and mono-unsaturated fatty acids has been shown to reduce the risk of cardiovascular disease (CVD) events in those at increased risk of CVD. ${ }^{(1,2)}$ In this study we assessed the association between three different dietary patterns measures and incident cardiovascular disease in the Scottish population. We used information on diet from the 1995, 1998, 2003, 2008 and 2010 Scottish Health Surveys, with food group and response categories regrouped where necessary to harmonize the data. A diet quality index (DQI), modified from one developed for the 2003 Scottish Health Survey ${ }^{(3)}$ (scoring intake of fruit and vegetables, fish, starchy foods, fibre, sugary and fatty foods and salt use) was calculated for each participant. Principal components analysis was used to identify a 'healthy' diet pattern (loading positively for wholemeal bread, fruit, high fibre breakfast cereals, vegetables, oily fish and potatoes, rice and pasta, and negatively for white bread, salt use and higher fat milk) and a 'traditional' diet pattern (loading positively for cakes and biscuits, confectionery, meat, potatoes rice and pasta and cheese), which accounted for $13 \%$ and $7 \%$ of the total variance in diet respectively. We included men and women aged 35y and over without CVD at the time of their survey measurements. Follow up of all participants to December 2012 was achieved by record linkage to the Scottish Morbidity Records, carried out by the Information Services Division of the Scottish Government. CVD events during follow up were defined as non-fatal hospital admissions or death coded with the International Classification of Disease revision 10 codes I20-25, I60-69 or G45. Odds of having a CVD event were assessed for each quintile of the DQI percentage score and the healthy diet and traditional diet patterns using logistic regression with adjustment for age and sex (model 1) or age, sex, BMI, smoking and socio-economic status (model 2). Among the 15,474 participants included in model 1, 1,848 had a recorded CVD event; model 2 included 13,762 participants of whom 1,626 had a CVD event.

\begin{tabular}{|c|c|c|c|c|}
\hline \multirow[b]{2}{*}{ Dietary pattern measure } & \multicolumn{2}{|c|}{ Model 1} & \multicolumn{2}{|c|}{ Model 2} \\
\hline & $\begin{array}{c}\text { OR }[95 \% \mathrm{CI}] \text { lowest } \\
\text { vs. highest quintile }\end{array}$ & $\mathrm{p}$ for linear trend & $\begin{array}{l}\text { OR }[95 \% \mathrm{CI}] \text { lowest } \\
\text { vs. highest quintile }\end{array}$ & $\mathrm{p}$ for linear trend \\
\hline DQI & $1.982[1.676,2.345]$ & $<0.001$ & $1.486[1.235,1.798]$ & $<0.001$ \\
\hline 'Traditional' diet pattern & $1.097[0.936,1.286]$ & 0.356 & $1.117[0.942,1.325]$ & 0.305 \\
\hline
\end{tabular}

Both the DQI and the healthy diet pattern showed clear associations with the odds of a CVD event which was raised by $49 \%$ in participants in the lowest quintile of the DQI percentage score and by $34 \%$ in participants in the lowest quintile of the healthy diet pattern compared with those in the highest quintiles in the fully adjusted models. The traditional diet pattern showed no significant association with incident CVD.

This work was supported by funding from the Scottish Government's Rural and Environment Science and Analytical Services (RESAS) division.

1. De Lorgeril M, Salen P, Martin JL et al. (1999) Mediterranean diet, traditional risk factors and the rate of cardiovascular complications after myocardial infarction: final report of the Lyon Diet Heart Study. Circulation 99, 779-85.

2. Estruch R, Ros E, Salas-Savadó J et al. (2013) Primary prevention of cardiovascular disease with a Mediterranean diet. $N$ Engl J Med 368, 1279-90.

3. Armstrong J, Sherriff A, Wrieden WL et al. (2009) Deriving and Interpreting Dietary Patterns in the Scottish Diet: Further Analysis of the Scottish Health Survey and the Expenditure and Food Survey. Food Standards Agency Scotland. http://www.foodstandards.gov.scot/deriving-andinterpreting-dietary-patterns-scottish-diet-further-analysis-scottish-health-survey-and 